\title{
Three-Dimensional Gaze Projection Heat-Mapping of Outdoor Mobile Eye-Tracking Data
}

\section{James Simpson*}

Lecturer

Department of Landscape Architecture

The University of Sheffield

j.c.simpson@sheffield.ac.uk

*corresponding author

\section{INTRODUCTION}

Advancements in neurological research provide new insights into the way routine urban experience can be considered as embodied (Mallgrave, 2013; 2015; 2018; Jelic et al., 2016; Huskinson, 2018). This builds upon the understanding that the central nervous system (or mind), physical body, and inhabited environment are holistically integrated, with individuals being active perceivers situated within the dynamics of their surroundings (Chiel \& Beer, 1997; Gallagher, 2005; Turner, 2017). The detailed analysis of embodied urban experience has recently been made possible through the mobilization of data collection methods for use in outdoor settings (Hein et al., 2008; Spinney, 2015). Such mobilization provides opportunity to track and analyze peoples' movement and activity within urban environments (Birenboim, 2018; Duchowny et al., 2018), as well as capture how they engage cognitively and perceive their surroundings (Gramann et al., 2011; Ladouce et al., 2017). The latter has been achieved through the use of techniques previously used within the laboratory during neuroscientific and cognitive science studies. These span in-the-field application of eye-tracking (Kiefer et al., 2017; Uttley et al., 2018), electroencephalography (EEG) (Mavros et al., 2016), and functional near-infrared spectroscopy (fNIRS) (Ladouce et al., 2017).

This study uses mobile eye-tracking in real-world urban streets. As a method, it provides insight into a person's distribution of gaze while offering an opportunity to infer what aspects of the inhabited environment capture their visual attention at a given point in time (Findlay and Gilchrist, 2003; Rothkopf et al., 2007). It is by no means a new data collection method, especially within controlled laboratory situations (Duchowski, 2017; Holmqvist et al., 2011). Detailed insight into the way people visually engage depictions of various outdoor urban scenes have emerged from lab studies (Emo, 2018; Hollander et al., 2019; Hollander et al., 2020; Noland et al., 2017). However,
Abstract /

The mobilization of eye-tracking for use outside of the laboratory provides new opportunities for the assessment of pedestrian visual engagement with their surroundings. However, the development of data representation techniques that visualize the dynamics of pedestrian gaze distribution upon the environment they are situated within remains limited. The current study addresses this through highlighting how mobile eye-tracking data, which captures where pedestrian gaze is focused upon buildings along urban street edges, can be mapped as three-dimensional gaze projection heat-maps. This data processing and visualization technique is assessed during the current study along with future opportunities and associated challenges discussed.

Keywords /

mobile eye-tracking; heat-map; gaze projection; urban street edge; embodied experience 
the ecological validity of these studies requires careful consideration, especially when seeking to generalize results with how people truly distribute their gaze in urban environments (Ladouce et al., 2017). This is a result of experimental stimuli not fully aligning with the immersive reality of real-world settings and the embodied nature of routine urban experience (Heft, 2019; Sun et al., 2018). Such caution is supported by Uttley et al.'s (2018) critique of eye-tracking methods, as well as Foulsham, Walker and Kingstone's (2011) finding that gaze behavior is significantly different between outdoor mobile situations and indoor laboratory contexts. These limitations have resulted in eye-tracking being used increasingly more during real-world situations (Kiefer et al., 2017; Uttley et al., 2018). Within an urban context, the technique has been used to assess pedestrian gaze behavior with other pedestrians (Fotios et al., 2015), understand how people visually engage with signage and facades of buildings (Tang, 2020), examine how tasks and differing street environments influence peoples' visual engagement with buildings (Simpson et al., 2019a), capture how visual engagement with street edges differs along non-pedestrianized and pedestrianized streets (Simpson et al., 2019b), show how people distribute their gaze when navigating paths and stairs (Marius't Hart \& Einhäuser, 2012), and use maps when wayfinding (Kiefer et al., 2014; Koletsis et al., 2017). Each of these studies have sought to situate participants within the shifting complexity of everyday urban settings.

Even though mobile eye-tracking is being used more frequently outdoors, there have been relatively few attempts to explore new ways of articulating the captured data. This is noticeable through the persistent use of data representation techniques that originated when insights were derived from static laboratory experiments (Uttley et al., 2018). The resulting modes of analysis and visualizations created lack responsiveness to the integrated influence of mind, dynamic body, and spatial richness of the built environment; by dint of their construction, they are simply unable to fully make visible peoples' embodied engagement with their surroundings. This investigation seeks to address this by exploring how mobile eye-tracking data capturing pedestrian visual engagement with buildings along urban street edges can be visualized as three-dimen- sional gaze projection heat-maps.

Heat-mapping is regularly employed as a technique for qualitatively visualizing eye-tracking data (Holmqvist et al., 2011). The approach graphically highlights how an individual or collective distribute their gaze, often upon two-dimensional stimuli. This then enables opportunity to assess where people predominantly focus their visual attention. As a technique, heatmaps "provide quick, very intuitive, and in some cases objective visual representations of eye-tracking data that naïve users and even children can immediately grasp a meaning from" (p. 231). This is important when producing data visualizations that are intended to be interpreted and used by individuals not trained in using eye-tracking. One group of potential users are built environment decision-makers, particularly those seeking to evidence design ideas and align interventions with how users routinely engage urban settings (Billger et al., 2017; Uttley et al., 2018). Building upon the established use of two-dimensional heat-mapping, recent lab-based experiments examined the potential of heat-mapping gaze distribution upon real and virtual three-dimensional objects (Alexiou et al., 2019; Li et al., 2019; Tang, 2020; Wang et al., 2018). These studies provide precedent necessary to explore three-dimensional heat-mapping using outdoor mobile eye-tracking data.

Alongside three-dimensional heat-mapping, there is opportunity to examine the way gaze is directed within three-dimensional space onto objects (i.e., from the eye of the perceiver to an aspect of the surrounding environment). This method has been previously employed during the mapping of gaze vectors to understand the distribution and angle of visual engagement in an indoor airport environment (Müller-Feldmeth et al., 2014; Schwarzkopf et al., 2017). There has been no use, however, of this technique within the dynamic complexity of outdoor urban situations, nor any attempt to combine an understanding of gaze projection in combination with heat-mapping. This investigation seeks to achieve this, and in doing so, aligns with recent developments in urban isovist and visibility analyses. Previous work in this area has started to explore weighted views through the assessment of viewer gaze projection upon a three-dimensional computerized scene and the subsequent influence of the stimuli on 
their perception (Fisher-Gewirtzman, 2018). Such research has also taken an embodied turn, with a distinct focus on the eye-level situated perceiver (Emo, 2015; Krukar et al., 2017) and the space-time dynamics and motion of engagement within a real-world environment (Fisher-Gewirtzman et al., 2003). These advancements show a clear desire to more thoroughly understand the combined influence that a dynamically situated perceiver and their surroundings have on real-world visual engagement.

This study builds upon previous mobile eye-tracking and data visualization methods during the production of three-dimensional gaze projection heat-maps. The produced mappings are reviewed in line with current street edge understandings to identify opportunities and challenges associated with such a data processing technique. Also undertaken is an assessment of the method's capacity to offer new knowledge that can inform how urban environments can be manipulated according to how people routinely visually engagement with them.

\section{METHODS}

\section{Participants}

24 participants took part in the study ( $\mathrm{n}=12$ female; $\mathrm{n}=12$ male; mean age $=35$ years; range $=21-61$ years). All were recruited via opportunity sampling, using a volunteers list managed by the University of Sheffield. All academic staff were removed from the participation invite and no participants were students from built environment design professions. This was to limit the influences education level and expertise might have upon visual engagement with the street environments. Study participants did not know the aim of the study prior to taking part, had normal to corrected-to-normal vision (through contact lenses), and prior experience walking on the selected study streets.

\section{Apparatus}

Mobile Eye-tracking Glasses (Glasses 2.0, SensoMotoric Instruments (SMI), Teltow, Germany) were used. These glasses contain three cameras that record the wearer's eye-movements individually and the environment to the front. The videos were processed in SMI BeGaze, creating a ten frame-per-second video. This consisted of gaze location, represented by a cross-hair, superimposed over a video of the environment being viewed. During data collection each participant wore a peaked cap to limit the impact that sunlight had on eye-tracking data quality, which is consistent with previous outdoor eye-tracking investigations (Kiefer et al., 2014). The lead researcher, wearing a small camera on their chest, followed each participant during data collection to record their location. This method was used due to issues with GPS accuracy identified during pilot studies.

\section{Study Procedure}

Before beginning their route, the eye-tracker was calibrated to the wearer with a three-point process; this was repeated until gaze tracking was accurate. Participants' eye-height was also measured. This information was used during the data mapping process. 
Once wearing the eye-tracker, each participant walked a short route around six streets in Sheffield, UK. In total, twelve study streets were used across two routes that were walked in the same direction (Streets 1-6 and Streets 7-12). Prior to stepping off along each street, participants were instructed to read a task card detailing a representative activity to undertake while walking. The activities were intended to give the study a greater level of real-world validity and were selected based upon on-site observation of pedestrian behaviors. Six activities were used, categorized by optional actions (breaktime stroll, coffee with a friend, window-shopping) and necessary actions (rushing to work, dropping off an object with a friend, walking to the bus). The use of these categorizations follows previous research (Gehl, 2010) and mobile eye-tracking studies (Simpson et al., 2019a). Such research showed that while optional and necessary activities influence the duration of street edge visual engagement, certain street edges are engaged with for longer periods, no matter the activity category.

The selected activities were dispersed across each of the six streets, meaning that each participant carried out each activity only once along their route. The overall intention was to expose participants to variable, real-world situations. No measures were taken to control any aspect of the environment, beyond the requirement to walk along specified streets in the same direction and undertake defined activities.

\section{Data Processing, Coding, and Visualization}

To select the data to be mapped, the eye-tracking videos were manually coded using VideoCoder (Foulsham et al., 2011). This output provided a gaze duration timeline, allowing insight into the amount of time participants visually engaged with buildings and other elements along the street edges of the 12 study streets. A subset of this coded eye-tracking data was then selected for visualization based upon which street edges were visually engaged with the most (Street 1: Chapel Walk and Street 2: Devonshire Street), the least (Street 5: Norfolk Street

1 and Street 6: Norfolk Street 2), and at the dataset's median (Street 3: Westfield Terrace and Street 4: Glossop Road). This subset provide a spectrum of eye-tracking data; Figure 1 highlights the pedestrian eye-level characteristics of the identified streets.

From this six-street data-subset, each participants' eye-tracking video was synchronized with the video from the chest camera worn by the lead researcher; this resulted in a video that highlighted both participant gaze (from the mobile eye-tracker) and body location (from the researcher worn camera). Information from this combined video was then mapped onto three-dimensional models of the study streets. Manual processing was necessarily employed because there is no effective automated system that can accurately interpret the complex three-dimensional dynamics of a pedestrian visually engaging with their surroundings.

The three-dimensional models of the study streets were produced using Trimble SketchUp; this modeling package was selected because of its ease of use and 


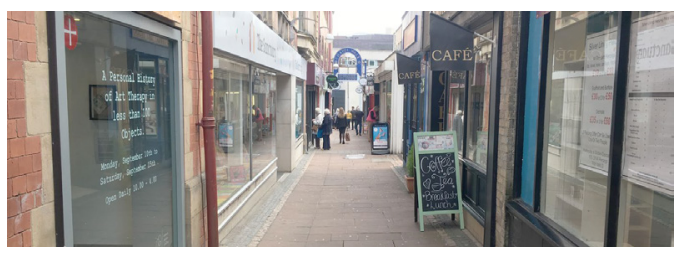

Figure 1 / Pedestrian eye-level images of selected streets

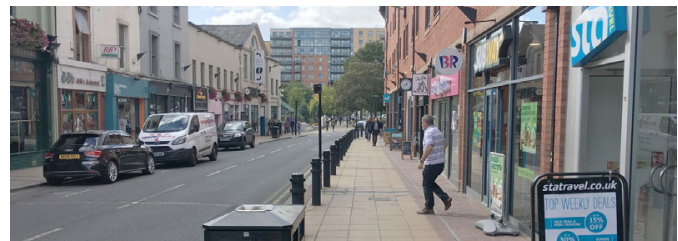

Most visually engaged street edges 2 , Street 2, Devonshire Street

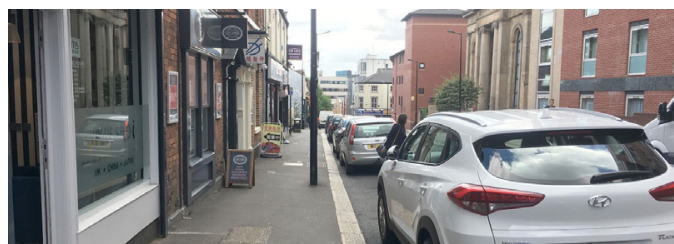

Median visually engaged street edges I, Street 3,Westfield Terrace

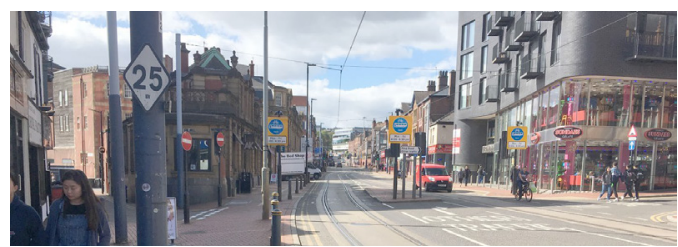

Median visually engaged street edges 2 Street 4, Glossop Road

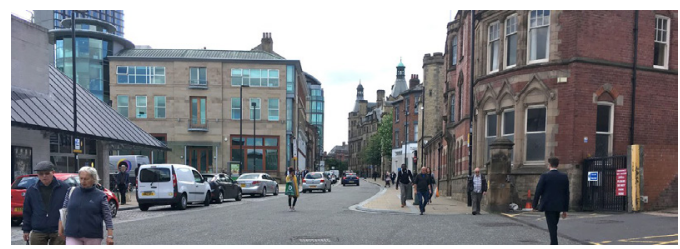

Least visually engaged street edges 1 . Street 5, Norfolk Row 1

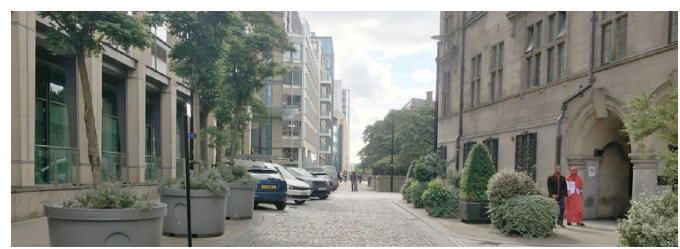

Least visually engaged street edges 2 Street 6, Norfolk Row 2

effectiveness when analyzing visibility of urban scenes (Lin et al., 2017). To three-dimensionally map the projection of each participant's gaze, from their eye location to the surrounding street edges, SketchUp was used in combination with the point editing plug-in Vertex Tools. Supplementary Figure 1 in Appendix I shows mapping examples for participants walking along Street 3 (Westfield Terrace). Gaze projection data for each participant was then overlaid for each street, producing combined three-dimensional mappings. The imagery for each street, was exported in plan-view and elevation for the left and right street edges. This process was required for the eye-tracking data to be effectively understood at the individual street scale. Supplementary Figure 2 in Appendix I provides example views of a combined three-dimensional mapping that incorporates all data for Street 3 (Westfield Terrace). Finally, the plan and elevation images were processed with Adobe Photoshop's gradient tool to produce the final heat-mappings. 


\section{Results and Discussion of Opportunities and Challenges}

The visualizations produced through the three-dimensional gaze projection heat-mapping of the mobile eye-tracking data can be seen in Figure 2. Larger scale mappings for each street can be found in the supplementary figures section (see Appendix I).

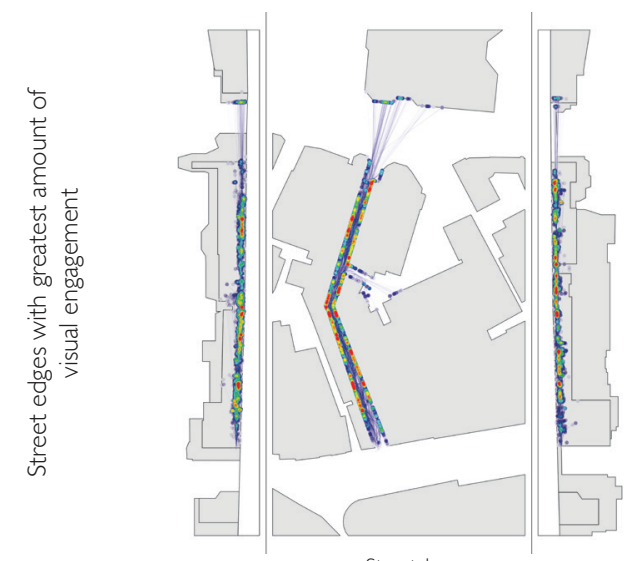

Street 1

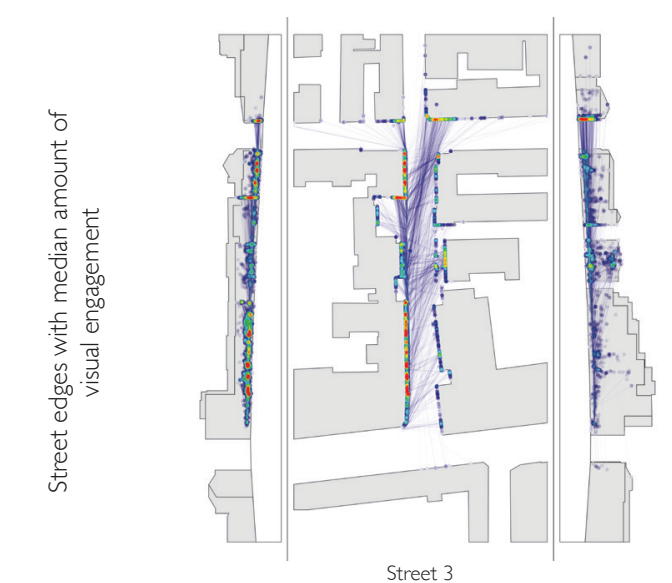

Street 3

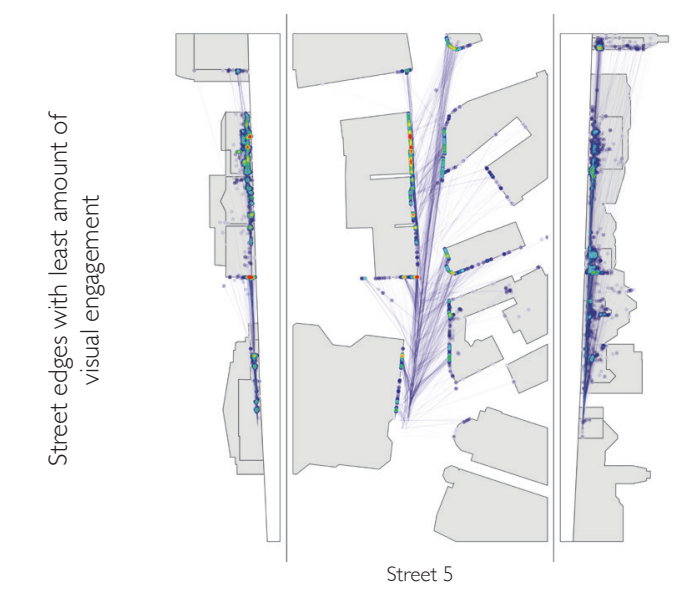

Heat-map key

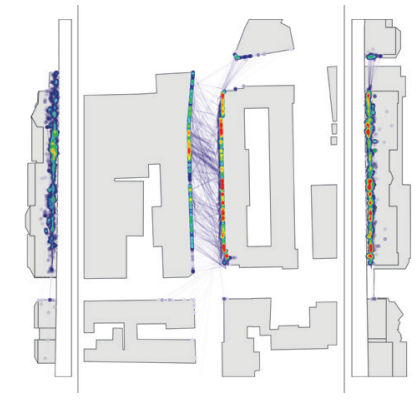

Figure 2 / Three-dimensional gaze projection heat-mapping of pedestrian visual engagement with urban street edges
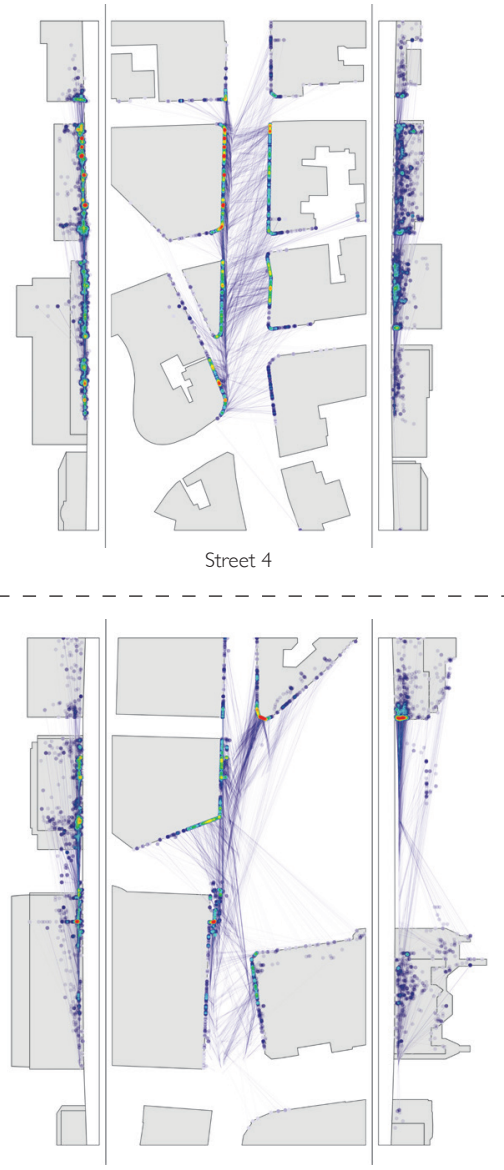

Street 6 
Figure 2 highlights collective pedestrian visual engagement with different street edges, captured while study participants were situated within the real-world dynamics of urban streets. A broad evaluation of the gaze behaviors, made visible by these mappings, follows. This is in order to better understand and provide a foundation from which to evaluate the effectiveness of the methods employed.

The greatest points of gaze focus were nearly always on the lower portions of the street edges. Even though this visual dominance is common across all the mappings, there is considerable variation between the different study streets. Nonetheless, this observation helps clarify just how disproportionately important building ground floors are at the point of pedestrian experience (Gehl et al., 2006; Karssenberg et al., 2016; Rahman \& Mehta, 2020), an aspect of streetscape experience that is often challenging to capture and articulate.

The mappings highlight how the most visually engaged street edges had a greater continuity of high intensity ground floor gaze focus along their length. The edges that were engaged with less had a greater proportion of gaze distributed across their entirety, including upper floors, with only two or three points of noticeably higher ground floor gaze focus. This insight correlates with the understanding that experientially engaging street edges have ground floors that capture and hold pedestrian attention more effectively along their extent, often through a rhythmic linear structure with numerous points of interest (Gehl et al., 2006; Hassan et al., 2019; Thwaites et al., 2020). The effect of this is heightened when pedestrians were actively undertaking the study's optional activities (breaktime stroll, coffee with a friend, window-shopping), as these actions naturally direct gaze towards ground floor shops and businesses (Simpson et al., 2019a). When street edges are structured to accommodate a finegrain, variable mix of these facilities along their length, (Streets 1 and 2, for example) these optional activities will naturally encourage significantly more ground floor visual engagement, as previously evaluated by Simpson et al. (2019b).

The visuals show how the linearity of streets predominantly directs gaze on the surrounding street edges in a forward direction aligned with the path of travel (study participants walked the streets from bottom to top of the mappings). Of this gaze, there is a dominant focus towards the street edge on the side being walked (see the right side of Street 2 and left side of Streets 3, 4 and 5). Streets 1 and 6 have a more balanced distribution of gaze, likely the result of pedestrianization. Although these observations can be evidenced quantitatively (Simpson et al., 2019b) and described via observations (Gehl et al., 2006), it had not been possible to visualize this phenomenon previously. The mapping processes employed during this study provides tangible, graphic insight into such pedestrian gaze behavior.

The most engaged street edges were along narrower, continuous streets with fewer edge breaks and setbacks. This type of environment seems to effectively contain visual engagement along the street edge, as more open streets had less intense edge engagement (see Streets 5 and 6). Such insight aligns with Thwaites et al. (2020) and Gehl's (2010) arguments that human scaled streets are important to stimulate and intensify pedestrian-environment interactions, particularly with street edges. Likewise, the mappings support the importance of morphological continuity when seeking to establish street edges that encourage active pedestrian engagement (Thwaites et al., 2020).

Interpreting the mappings produced in line with existing street edge knowledge provides insight into the analytical capabilities of the developed techniques. There are, of course, opportunities and challenges associated with the production of three-dimensional gaze projection heat-maps along with the use of mobile eye-tracking outdoors, which require deeper evaluation.

\section{Opportunities and Challenges}

Mobile eye-tracking in real-world urban situations provides insights that are more ecologically valid and aligned with realistic visual experiences than those in laboratory contexts (Ladouce et al., 2017; Uttley et al., 2018). This is significant, considering the pressing need for representative empirical insight that can be used to effectively inform design decision-making (Simpson et al., 2019a; Uttley et al., 2018). Many have questioned the knowledge foundations from 
which built environment design intervention is based (Cuthbert, 2007; Marshall, 2012; Mehta, 2013). Through mobile eye-tracking there is an opportunity to address this by establishing a rigorous practice around the structure of pedestrian visual experience. The result is new opportunity to guide decision-making and bring design interventions closer to how people routinely engage with urban spaces.

The format of insights and the way in which they are articulated are equally significant. The approach developed seeks to visualize gaze information in a manner that is comprehensible without the need for a detailed understanding of eye-tracking metrics or scientific analyses. The outcome is an innovative communication tool that can readily explain the complexities of pedestrian visual experience and be used to directly inform environmental change approaches. Linked with this is the how the data visualization method developed has the potential to provide generalizable insights. This has been shown through its capacity to complement and evidence, in a visually tangible manner, existing street edge knowledge. There is also, however, an opportunity to provide context and site-specific insights into gaze behavior. For example, along Street 2 there was a noticeable focus of pedestrian gaze towards a specific area of the opposite sided street edge. This was the site of a new shop that clearly grabbed the visual attention of participants, as it was a previously un-experienced aspect of the environment. The heat maps produced showed the experiential influence of this small-scale environmental change that might otherwise have evaded elicitation. This potential for both broad and contextualized, insight from a single tool offers clear opportunities for informing design across different scales. There is opportunity to evidence and guide, through making comprehensible trends in human-environment interactions, how future built environment interventions are approached. Likewise, at a context specific scale, the technique has the potential to highlight areas that require focused design attention to encourage more experientially engaging urban settings.

To date, eye-tracking analysis has been predominantly used to provide insights into the distribution of peoples' gaze upon a given stimulus by categorizing and measuring eye-movements (Duchowski, 2017; Holmqvist et al., 2011). However, the data processing and visualization techniques developed offer the potential to combine an understanding of eyemovements with a broader physiological analysis of how the human body is situated, orientated, and moving within an environment. Previous eye-tracking research has examined the horizontal angle of gaze projection indoors (Müller-Feldmeth et al., 2014; Schwarzkopf et al., 2017) and the way surface complexity influences eye and head angle has been explored (Thomas et al., 2020). This study builds upon such work within a mobile outdoor context and in doing so, there is clear potential for not only comprehending what people predominantly visually engage with (duration) but also the distance over which this engagement is projected and the angle at which it takes place in relation to the space inhabited (i.e., against the dominant forward focused linearity of a street). Constructing such an integrated understanding is significant, as both distance (Hall, 1966; Lynch \& Hack, 1984; Morello \& Ratti, 2009; Gehl, 2010) and the orientation of the body and eyes (FisherGewirtzman and Wagner, 2003; Gehl et al., 2006; Gibson, 1979; Yang et al., 2007) influence engagement with and perception of urban environments. As a result, this research provides opportunity to integrate eyetracking with recent developments in the isovist and visibility analysis of urban environments. However, further research is clearly needed in order for such advancements to be realized. Nonetheless, the current investigation has highlighted unrealized opportunities for combined research techniques through encouraging a more embodied and spatially responsive analysis of mobile eye-tracking data that seeks to comprehensively understand the duration, distance, and angle of situated human gaze.

It has been previously highlighted that there is opportunity to link eye-tracking with additional auxiliary data collection techniques (Holmqvist et al., 2011). Recently, researchers have sought to combine EEG (Ladouce et al., 2017), skin-conductance (Uttley et al., 2016), and verbal descriptions (Uttley et al., 2018) with outdoor mobile eye-tracking data, however, further technological developments are needed to effectively link these different data streams (Ladouce et al., 2017; 
Mavros et al., 2016). Although this is the present case, there is clear potential for the three-dimensional heatmapping method to incorporate additional sources of data. This would allow for greater links between gaze distribution and visual attention to be made, which is an ongoing challenge (Uttley et al., 2018). It would also offer opportunity to comprehend the embodied nature of complex urban experiences by linking different information sources that capture how the human mind and body react to the surrounding environment. Such insight could be used to inform evidence-based environmental changes that incorporate multiple, overlaid empirical sources of experiential information.

As described, mobile eye-tracking in real-world situations often provides insights that are more ecologically valid when compared against lab-based studies. When undertaking eye-tracking research in complex outdoor situations, however, it is challenging to control the inherent variability of the stimuli which study participants engage (Uttley et al., 2018). The resulting data is sometimes challenging to make direct comparisons across and draw substantive conclusions from. Further, when people are situated within outdoor settings such as streets, it can be difficult to assess if their attention is actually directed towards what they are looking at within a shifting and multisensory environment; these environments heighten the potential for people to be thinking about other aspects of their surroundings or previous experiences rather than what their eyes are directed towards (Hausdorff et al., 2005; Uttley et al., 2018). By layering and heat-mapping the projection of gaze this issue is mitigated through the cumulative aggregation of gaze data. There is future potential for such an issue to be overcome by integrating mobile eye-tracking and wider data collection techniques, as mentioned earlier. This triangulation of data would help establish more representative three-dimensional mappings of visual attention, that is what someone is seeing (cognitively processing) rather than just looking at (gaze). Virtual reality eye-tracking is also a potential technique that could address such issues through its ability to systematically control the stimuli exposed to study participants. Implementing this technique would also eliminate the need for a researcher to follow study participants, which was required for this study and might have influenced their visual behavior. However, the use of virtual reality eye-tracking requires further evaluation, as the immersive research stimuli is still an abstract version of the richness embodied in realworld situations.

This study involved extensive manual data processing. This encompassed categorization coding, using VideoCoder, and creating three-dimensional gaze maps with Trimble SketchUp and the Vertex Tools plug-in. These processes are labor and time intensive and are susceptible to human error, as frame-byframe interpretation and coding of eye-tracker videos is necessary. There is potential for these processes to be streamlined and automated, particularly as technological advancements are made in machine learning and the categorization of visual stimuli attributes (Badrinarayanan et al., 2017; Middel et al., 2019). Process automation within GIS and MATLAB softwares have been shown to be effective for threedimensional isovist analyses (Morello and Ratti, 2009; Yang et al., 2007). Similarly, virtual reality could offer opportunities for reducing data collection and coding workloads (Pfeiffer \& Memili, 2016; Uttley et al., 2018). Such advancements would reduce the effort and time needed to generate the visualizations while lessening the potential for human error associated with their production.

There are issues with research scale when using mobile eye-tracking outdoors (Uttley et al., 2018). As discussed, data capture, coding, and visualization is time-consuming, therefore limiting the amount of data that can be effectively processed and subsequently restricting widespread application and use. Despite this, there is opportunity to use the insights obtained during this study in combination with and to inform wider analytical approaches. For example, recent advancements in machine learning have allowed the large scale analysis of spatial and material attributes of urban streets that affect visual quality (Ye et al., 2019). Agent-based models have been used to assess human movement and behavior in combination with visual affordance (Turner, 2017; Turner \& Penn, 2002). Mobile eye-tracking, alongside three-dimensional mapping, could be used to complement and refine findings derived through these techniques. As a result, there 
is potential to scale-up eye-tracking insights to align innovative large-scale analyses with a detailed, embodied understandings of real-world pedestrian visual experiences.

\section{CONCLUSION}

The current study advances methods for articulating how people visually engage with urban spaces. The techniques are responsive to the integrated influence of human body, mind, and surrounding environment on peoples' routine experiences. This was achieved through three-dimensionally heat-mapping the projection of pedestrian gaze upon the occupied environment. Such a method advances existing techniques for evaluating outdoor mobile eye-tracking data, which have so far lacked sensitivity to the situated and embodied nature of dynamic visual engagement within urban environments. 


\section{REFERENCES}

Alexiou, E., Xu, P., \& Ebrahimi, T. (2019). Towards modelling of visual saliency in point clouds for immersive applications. 26th IEEE International Conference on Image Processing (ICIP). Taipei, Taiwan.

Badrinarayanan, V., Kendall, A. \& Cipolla, R. (2017). SegNet: A deep convolutional encoder-decoder architecture for image segmentation. IEEE Transactions on Pattern Analysis and Machine Intelligence, 39(12), 2481-2495.

Billger, M., Thuvander, L. \& Wästberg, B. S. (2017). In search of visualization challenges: The development and implementation of visualization tools for supporting dialogue in urban planning processes. Environment and Planning B: Urban Analytics and City Science, 44(6), 1012-1035.

Birenboim, A. (2018). The influence of urban environments on our subjective momentary experiences. Environment and Planning B: Urban Analytics and City Science, 45(5), 915-932.

Chiel, H. J. \& Beer, R. D. (1997). The brain has a body: Adaptive behavior emerges from interactions of nervous system, body and environment. Trends in Neurosciences, 20(12), 553-557.

Cuthbert, A. R. (2007). Urban design: Requiem for an era-review and critique of the last 50 years. Urban Design International, 12(4), 177-223.

Duchowny, K., Clarke, P., Gallagher, N. A., et al. (2018). Using mobile, wearable, technology to understand the role of built environment demand for outdoor mobility. Environment and Behavior, 51(6), 671-688.

Duchowski, A. T. (2017). Eye Tracking Methodology: Theory and Practice. Springer.

Emo, B. (2015). Exploring isovists: The egocentric perspective. 10th International Space Syntax Symposium (SSS). London.

Emo, B. (2018). Choice zones: Architecturally relevant areas of interest. Spatial Cognition \& Computation, 18(3), 173-193.

Findlay, J. M. \& Gilchrist, I. D. (2003). Active vision: The Psychology of Looking and Seeing. Oxford UP.

Fisher-Gewirtzman, D. (2018). Integrating 'weighted views' to quantitative 3D visibility analysis as a predictive tool for perception of space. Environment and Planning B: Urban Analytics and City Science, 45(2), 345-366.

Fisher-Gewirtzman, D., Burt, M., \& Tzamir, Y. (2003). A 3-D visual method for comparative evaluation of dense built-up environments. Environment and Planning B: Planning and Design, 30 (4), 575-587.

Fisher-Gewirtzman, D. \& Wagner, I. A. (2003). Spatial openness as a practical metric for evaluating built-up environments. Environment and Planning B: Planning and Design, 30 (1), 37-49.

Fotios, S., Uttley, J., \& Yang, B. (2015). Using eye-tracking to identify pedestrians' critical visual tasks. Part 2. Fixation on pedestrians. Lighting Research \& Technology, 47 (2), 149-160.

Foulsham, T., Walker, E., \& Kingstone, A. (2011). The where, what and when of gaze allocation in the lab and the natural environment. Vision Research, 51 (17), 1920-1931.

Gallagher, S. (2005). How the Body Shapes the Mind. Oxford UP.

Gehl, J. (2010). Cities for People. Island Press.

Gehl, J., Kaefer, L. J., \& Reigstad, S. (2006). Close encounters with buildings. Urban Design International, 11 (1), 29-47.

Gibson, J. (1979). The Ecological Approach to Visual Perception. Houghton Mifflin.

Gramann, K., Gwin, J. T., Ferris, D. P., et al. (2011). Cognition in action: Imaging brain/body dynamics in mobile humans. Reviews in the Neurosciences, 22(6), 593-608.

Hall, E. T. (1966). The Hidden Dimension. Doubleday.

Hassan, D. M., Moustafa, Y. M., \& El-Fiki, S. M. (2019). Ground-floor façade design and staying activity patterns on the sidewalk: A case study in the Korba area of Heliopolis, Cairo, Egypt. Ain Shams Engineering Journal, 10 (3), 453-461.

Hausdorff, J. M., Yogev, G., Springer, S., et al. (2005). Walking is more like catching than tapping: Gait in the elderly as a complex cognitive task. Experimental Brain Research, 164(4), 541-548.

Heft, H. (2019). What's wrong with using photographs of the environment in environmental perception research? 20th Environmental Design Research Association (EDRA) Conference. Brooklyn, NY.

Hein, J. R., Evans, J., \& Jones, P. (2008). Mobile methodologies: Theory, technology and practice. Geography Compass, 2 (5), 1266-1285.

Hollander, J. B., Purdy, A., Wiley, A., et al. (2019) Seeing the city: Using eye-tracking technology to explore cognitive responses to the built environment. Journal of Urbanism: International Research on Placemaking and Urban Sustainability, 12(2), 156-171. 
Hollander, J. B., Sussman, A., Purdy, A., et al. (2020). Using eye-tracking to understand human responses to traditional neighborhood designs. Planning Practice \& Research, 35(5), 1-25.

Holmqvist, K., Nyström, M., Andersson, R., et al. (2011). Eye Tracking: A Comprehensive Guide to Methods and Measures. Oxford UP. Huskinson, L. (2018). Architecture and the Mimetic Self: A Psychoanalytic Study of How Buildings Make and Break our Lives. Routledge.

Jelic, A., Tieri, G., De Matteis, F., et al. (2016). The enactive approach to architectural experience: A neurophysiological perspective on embodiment, motivation, and affordances. Frontiers in Psychology, 7, 1-20.

Karssenberg, H, Laven J, Glaser M, et al. (2016). The City at Eye Level: Lessons for Street Plinths.

Kiefer, P., Giannopoulos, I., \& Raubal, M. (2014). Where am I? Investigating map matching during self-localization with mobile eye tracking in an urban environment. Transactions in GIS, 18(5), 660-686.

Kiefer, P., Giannopoulos, I., \& Raubal, M., et al. (2017). Eye tracking for spatial research: Cognition, computation, challenges. Spatial Cognition \& Computation, 17(1-2), 1-19.

Koletsis, E., van Elzakker, C. P. J. M., Kraak, M-J., et al. (2017). An investigation into challenges experienced when route planning, navigating and wayfinding. International Journal of Cartography, 3(1), 4-18.

Krukar, J., Schultz, C., \& Bhatt, M. (2017). Towards embodied 3d isovists: Incorporating cognitively-motivated semantics of 'space’ and the architectural environment in 3D visibility analysis. 11th International Space Syntax Symposium (SSS). Lisbon, Portugal.

Ladouce, S., Donaldson, D. I., Dudchenko, P. A., et al. (2017). Understanding minds in real-world environments: Toward a mobile cognition approach. Frontiers in Human Neuroscience 10, 1-14.

Li, X., Yu, L., Fu, C-W., et al. (2019). Unsupervised detection of distinctive regions on 3D shapes. CoRR, 1(1), 1-16.

Lin, T., Lin. H., \& Hu, M. (2017). Three-dimensional visibility analysis and visual quality computation for urban open spaces aided by Google SketchUp and WebGIS. Environment and Planning B: Urban Analytics and City Science, 44 (4), 618-646.

Lynch, K. \& Hack, G. (1984). Site Planning. MIT press.

Mallgrave, H. F. (2013). Architecture and Embodiment: The Implications of the New Sciences and Humanities for Design. Routledge.

Mallgrave, H. F. (2015). Embodiment and enculturation: The future of architectural design. Frontiers in Psychology 6: 1-6.

Mallgrave, H. F. (2018). From Object to Experience: The New Culture of Architectural Design. Bloomsbury Publishing.

Marius't Hart, B. \& Einhäuser, W. (2012). Mind the step: Complementary effects of an implicit task on eye and head movements in reallife gaze allocation. Experimental Brain Research, 223 (2), 233-249.

Marshall, S. (2012). Science, pseudo-science and urban design. Urban Design International, 17 (4), $257-271$.

Mavros, P., Austwick M. Z., \& Smith A. H. (2016). Geo-EEG: Towards the use of EEG in the study of urban behaviour. Applied Spatial Analysis and Policy, 9(2), 191-212.

Mehta, V. (2013). The Street: A Quintessential Social Public Space. Routledge.

Middel, A., Lukasczyk, J., Zakrzewski, S., et al. (2019). Urban form and composition of street canyons: A human-centric big data and deep learning approach. Landscape and Urban Planning 183, 122-132.

Morello, E. \& Ratti, C. (2009). A digital image of the city: 3D isovists in Lynch's urban analysis. Environment and Planning B: Planning and Design, 36(5), 837-853.

Müller-Feldmeth, D., Schwarzkopf, S., Büchner, S. J., et al. (2014). Location dependent fixation analysis with sight vectors: Locomotion as a challenge in mobile eye tracking. 2nd International Workshop on Eye Tracking for Spatial Research (ET4S). Vienna, Austria.

Noland, R. B., Weiner, M. D., Gao, D., et al. (2017). Eye-tracking technology, visual preference surveys, and urban design: Preliminary evidence of an effective methodology. Journal of Urbanism: International Research on Placemaking and Urban Sustainability, 10 (1), 98-110.

Pfeiffer, T. \& Memili, C. (2016) Model-based real-time visualization of realistic three-dimensional heat maps for mobile eye tracking and eye tracking in virtual reality. Proceedings of the Ninth Biennial ACM Symposium on Eye Tracking Research \& Applications, 95-102.

Rahman, M. N. \& Mehta, V. (2020) Signage Form and Character: a window to neighborhood visual identity. Interdisciplinary Journal of Signage and Wayfinding, 4(1), 35-48.

Rothkopf, C. A., Ballard, D. H. \& Hayhoe, M. M. (2007). Task and context determine where you look. Journal of Vision, 7(14), 1-20.

Schwarzkopf, S., Büchner, S. J., Hölscher, C., et al. (2017). Perspective tracking in the real world: Gaze angle analysis in a collaborative wayfinding task. Spatial Cognition \& Computation, 17(1-2), 143-162. 
Simpson, J., Freeth, M., Simpson, K. J., et al. (2019a). Visual engagement with urban street edges: Insights using mobile eye-tracking. Journal of Urbanism: International Research on Placemaking and Urban Sustainability, 12(3), 259-278.

Simpson, J., Thwaites, K., \& Freeth, M. (2019b). Understanding Visual Engagement with Urban Street Edges along Non-Pedestrianised and Pedestrianised Streets Using Mobile Eye-Tracking. Sustainability, 11(15), 4251.

Spinney, J. (2015). Close encounters? Mobile methods, (post)phenomenology and affect. Cultural Geographies, 22(2), $231-246$.

Sun, M., Herrup, K., Shi, B., et al. (2018). Changes in visual interaction: Viewing a Japanese garden directly, through glass or as a projected image. Journal of Environmental Psychology, 60, 116-121.

Tang, M. (2020). Analysis of Signage Using Eye-Tracking Technology. Interdisciplinary Journal of Signage and Wayfinding, 4(1), 61-72.

Thomas, N. D. A., Gardiner, J. D., Crompton, R. H., et al. (2020). Look out: An exploratory study assessing how gaze (eye angle and head angle) and gait speed are influenced by surface complexity. PeerJ, 8, 1-13.

Thwaites, K., Simpson, J., \& Simkins, I. (2020). Transitional edges: A conceptual framework for socio-spatial understanding of urban street edges. Urban Design International, 25, 295-309.

Turner, A. (2017). Partners in the street ballet: An embodied process of person-space coupling in the built environment. Environment and Planning B: Urban Analytics and City Science, 44(2), 294-307.

Turner, A. \& Penn, A. (2002). Encoding natural movement as an agent-based system: An investigation into human pedestrian behaviour in the built environment. Environment and Planning B: Planning and Design, 29(4), 473-490.

Uttley, J., Fotios, S., \& Cheal, C. (2016). Measuring perceived safety after-dark - three alternatives to rating scales. 24th International Association of People-Environment Studies (IAPS) Conference. Lund, Sweden.

Uttley, J., Simpson, J., \& Qasem, H. (2018). Eye-tracking in the real world: Insights about the urban environment. In: Handbook of Research on Perception-Driven Approaches to Urban Assessment and Design. IGI Global.

Wang, X., Koch, S., Holmqvist, K., et al. (2018). Tracking the gaze on objects in 3D: How do people really look at the bunny? ACM Transactions on Graphics, 37(6), 1-18.

Yang, P. P-J., Putra S. Y., \& Li, W. (2007). Viewsphere: A GIS-based 3D visibility analysis for urban design evaluation. Environment and Planning B: Planning and Design, 34(6), 971-992.

Ye Y., Zeng W., Shen Q, et al. (2019) The visual quality of streets: A human-centred continuous measurement based on machine learning algorithms and street view images. Environment and Planning B: Urban Analytics and City Science 46(8), 1439-1457. 


\section{APPENDIX I: SUPPLEMENTARY FIGURES}
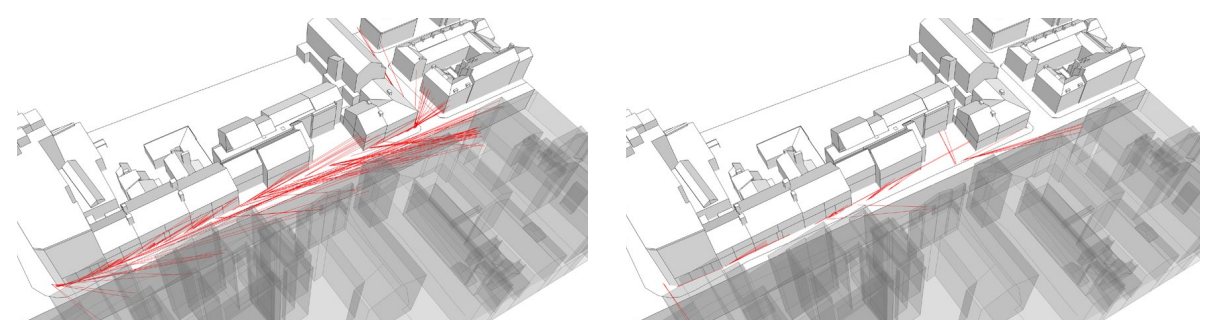

Necessary

Activity I Rushing to work
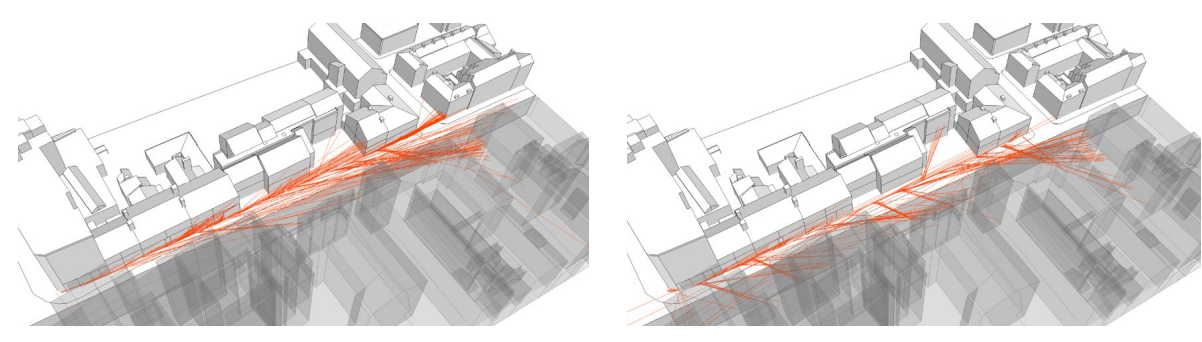

Necessary Activity 2 -

Dropping off

object with a friend
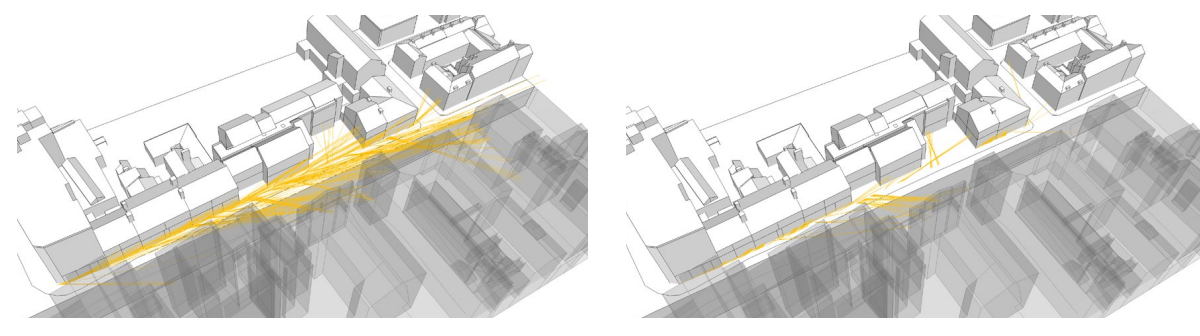

Necessary

Activity 3 -

Walking to the bus
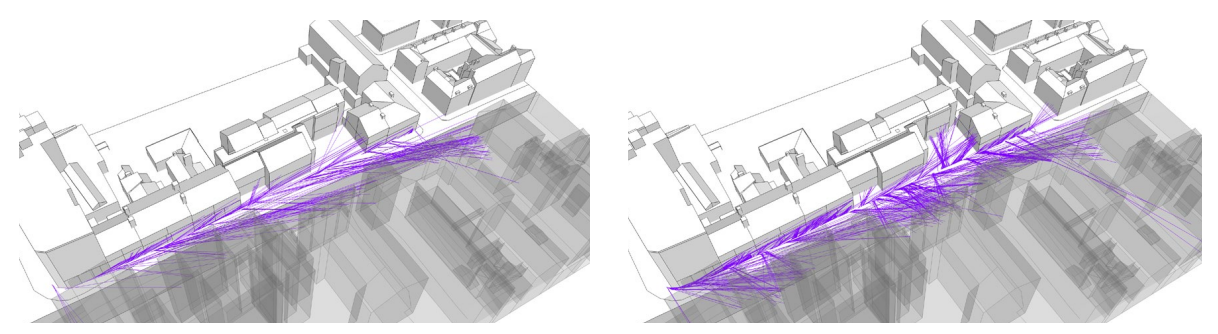

Optional

Activity I -

Breaktime stroll
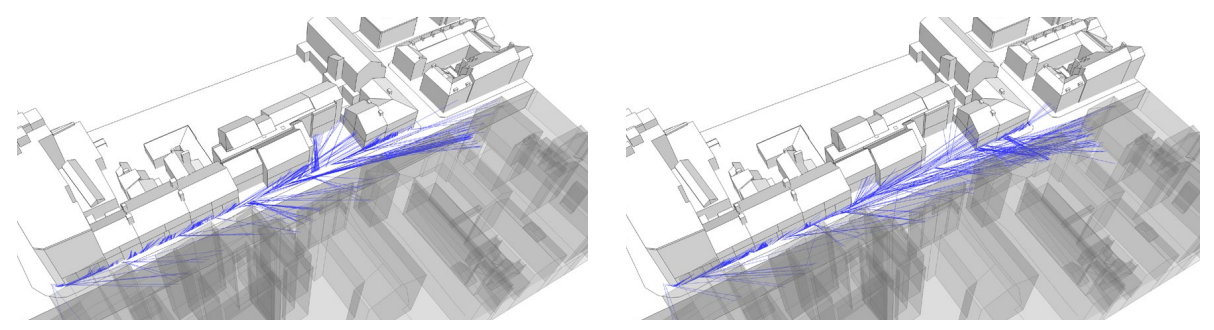

Optional

Activity 2

Coffee with

a friend
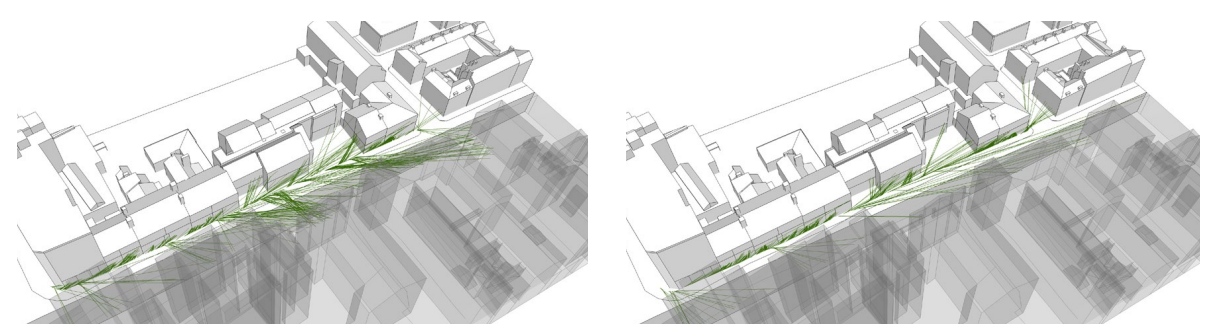

Optional Activity 3 Window-shopping

Supplementary Figure 1 / Three-dimensional mapping of individual study participant gaze along Street 3 

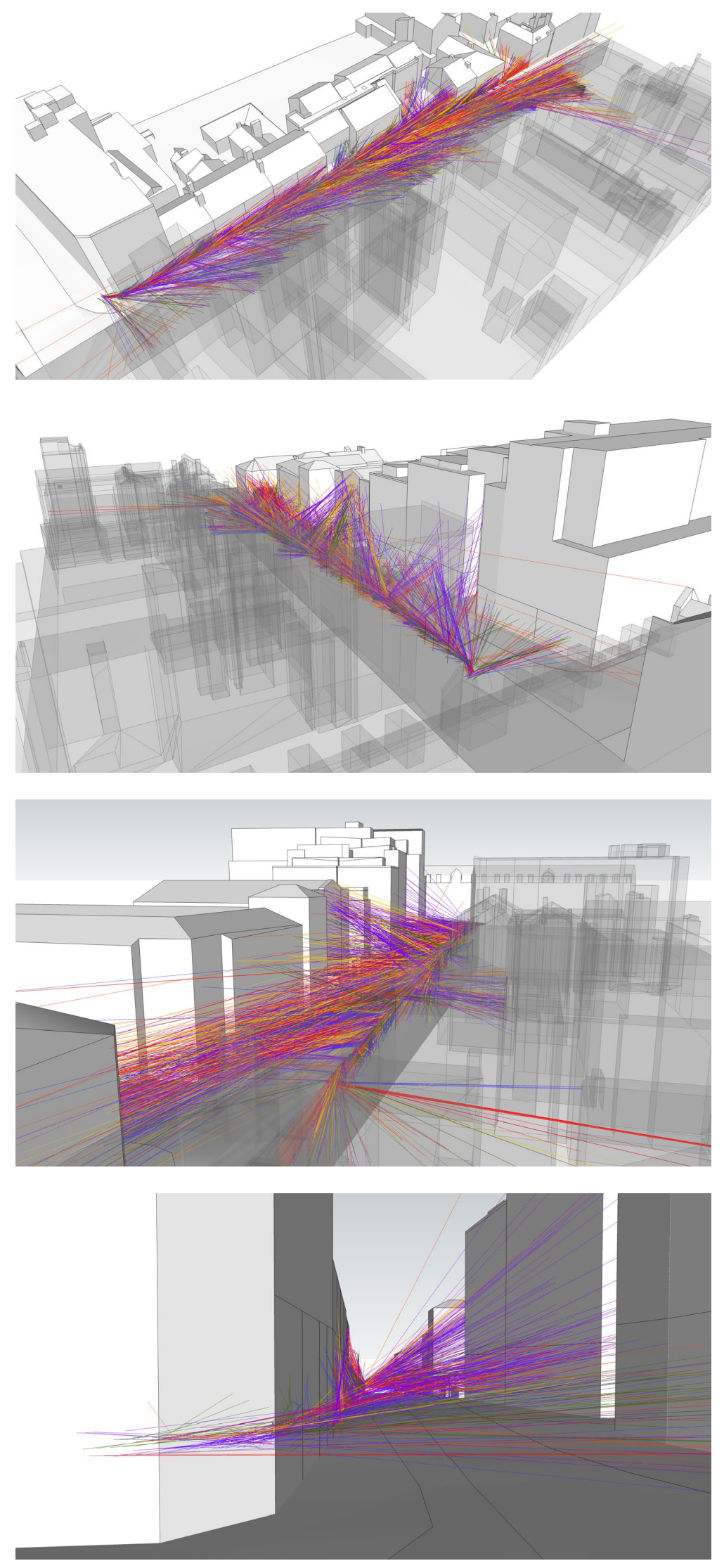

Supplementary Figure 2 / Combined three-dimensional mapping of gaze along Street 3 


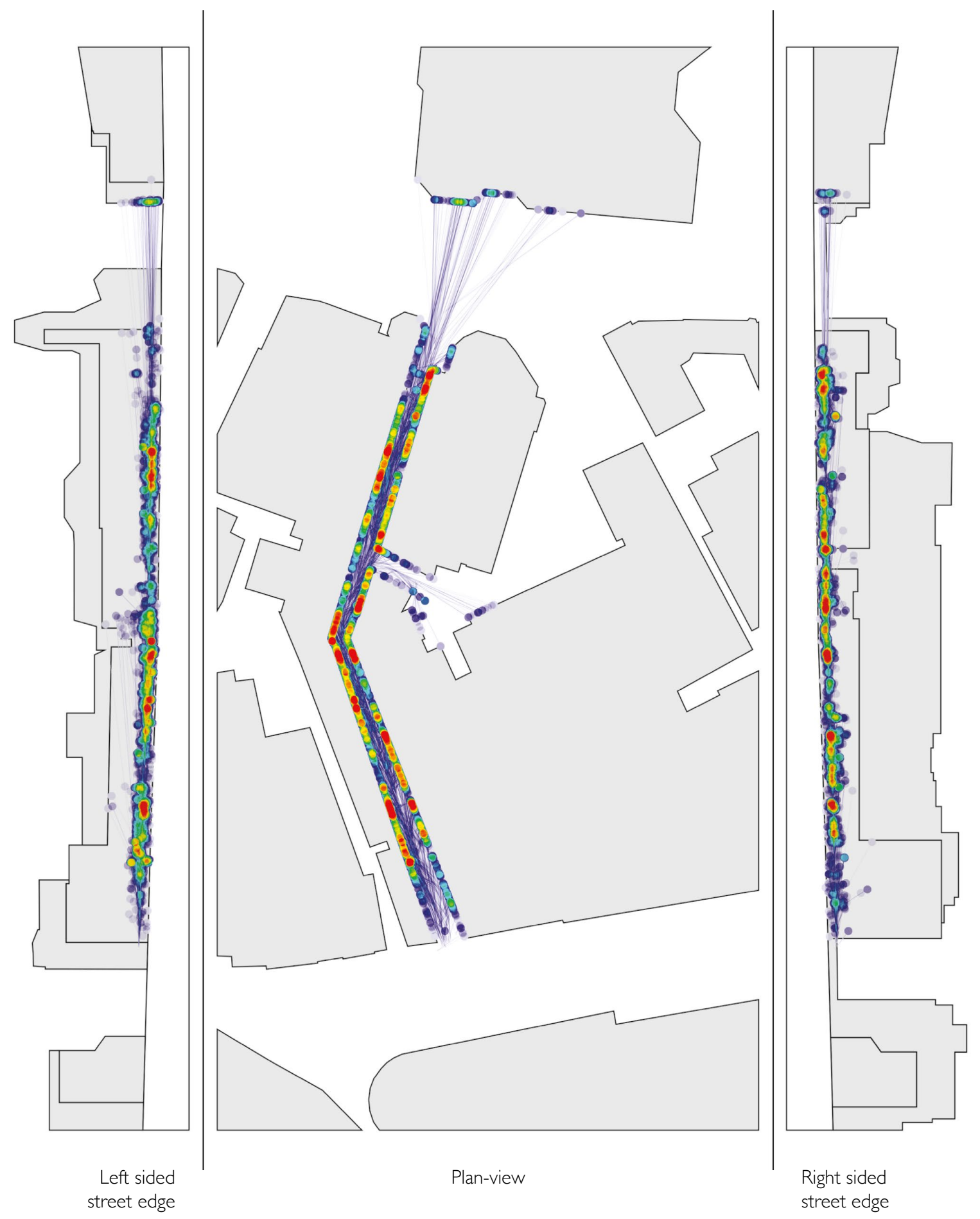

Heat-map key 


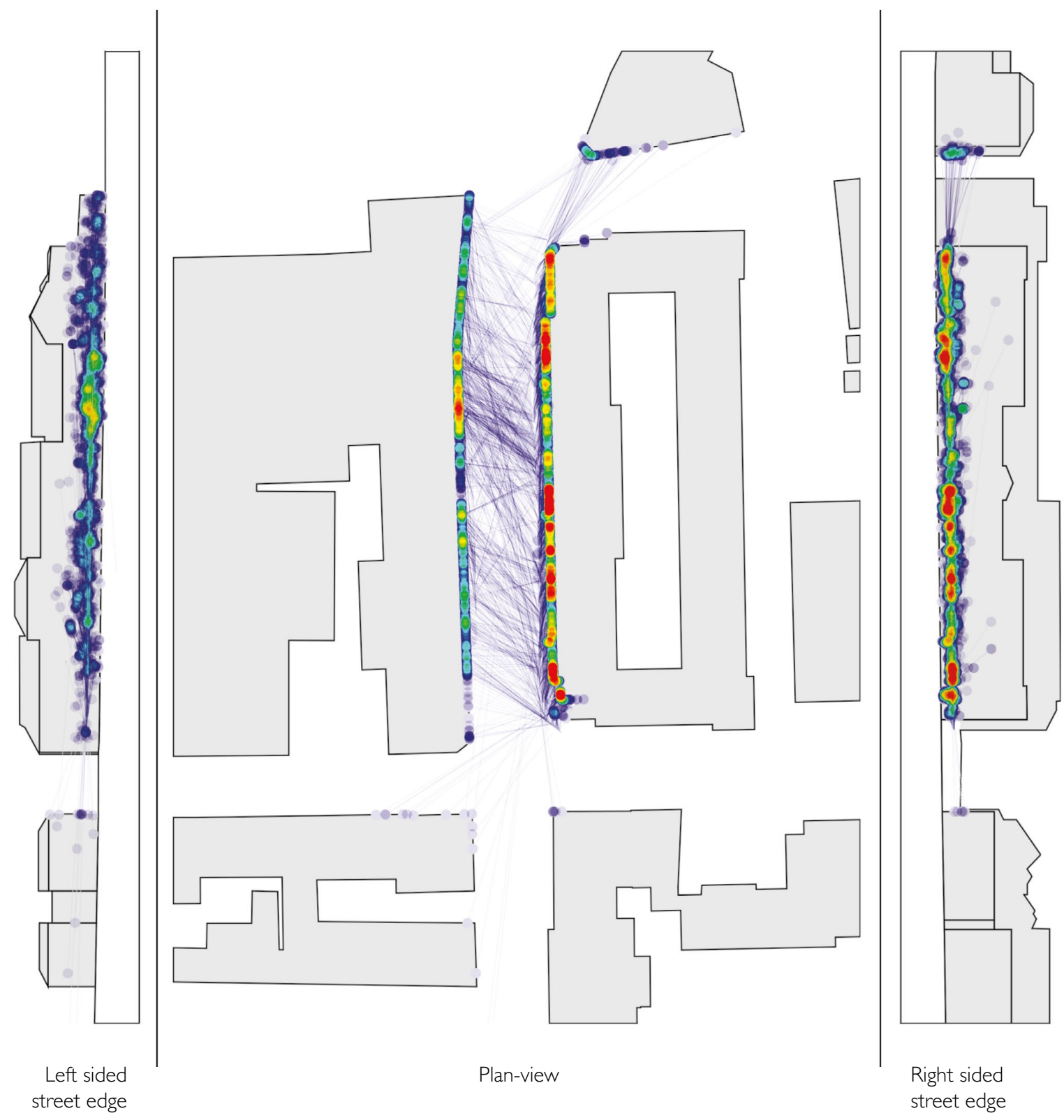

Heat-map key 

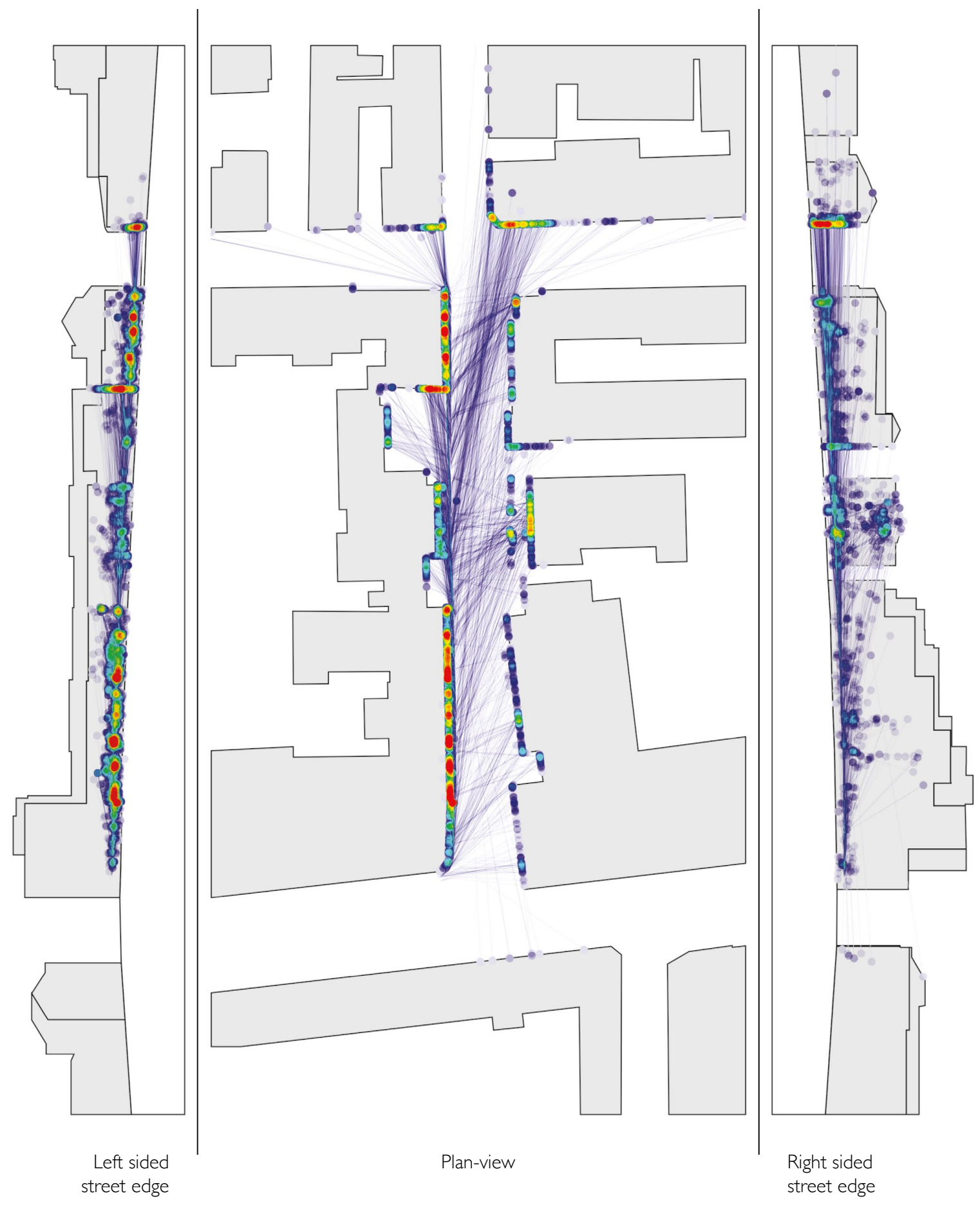

Heat-map key

Low amount of collective gaze

focus upon street edge

High amount of collective gaze

focus upon street edge

Supplementary Figure 5 / Street 3 mapping 


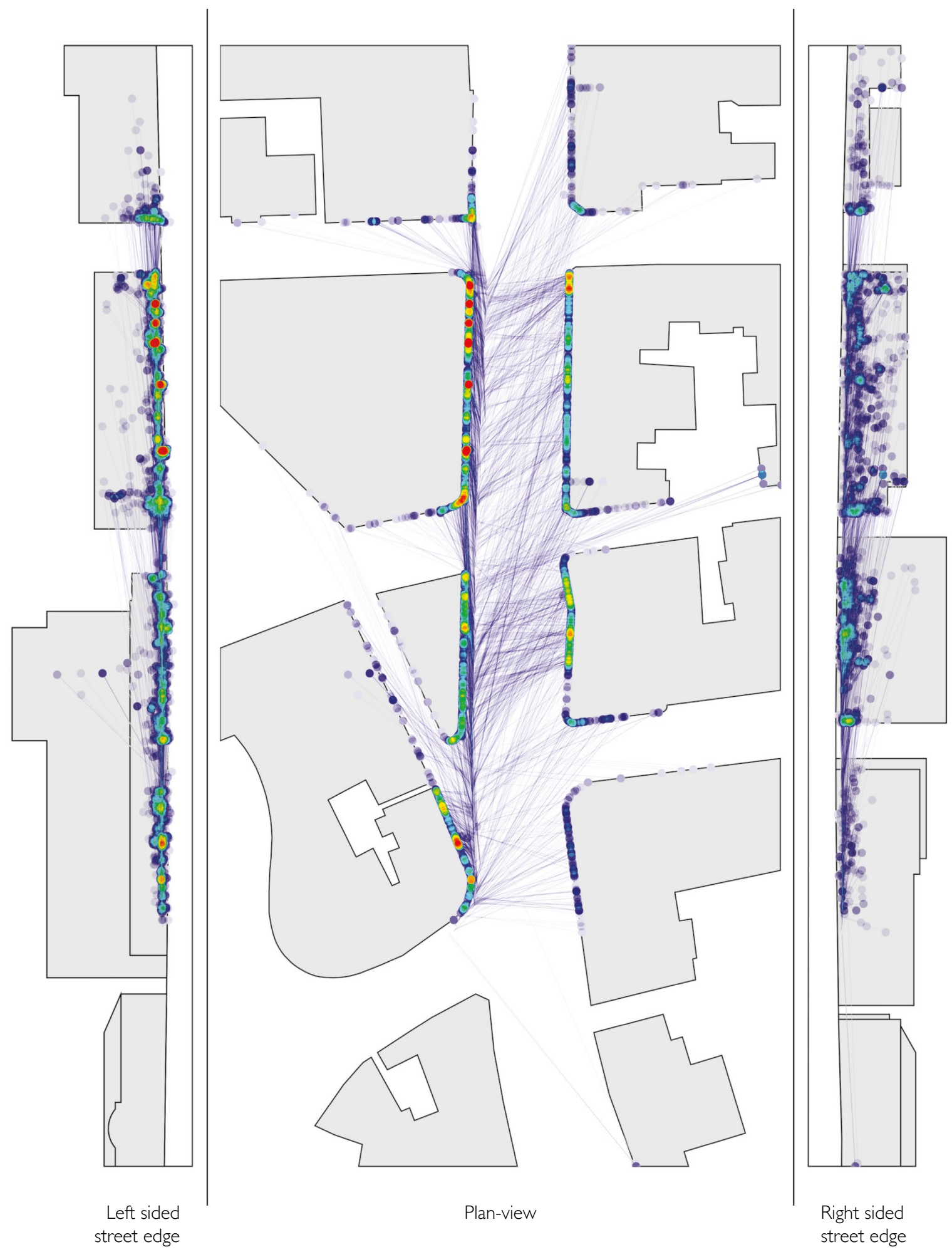

Heat-map key 


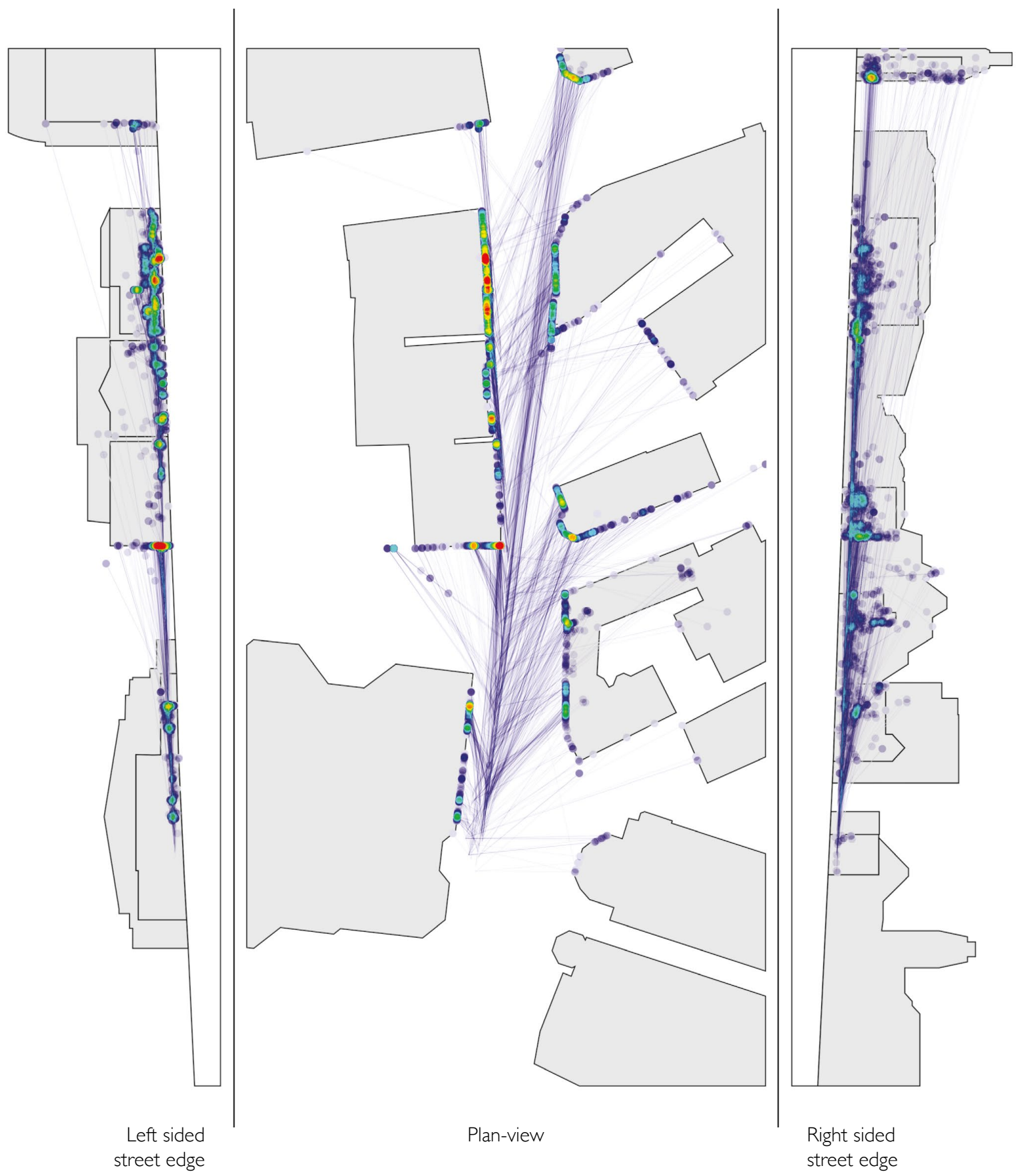

Heat-map key

High amount of collective gaze focus upon street edge

focus upon street edge

Supplementary Figure 7 / Street 5 mapping 


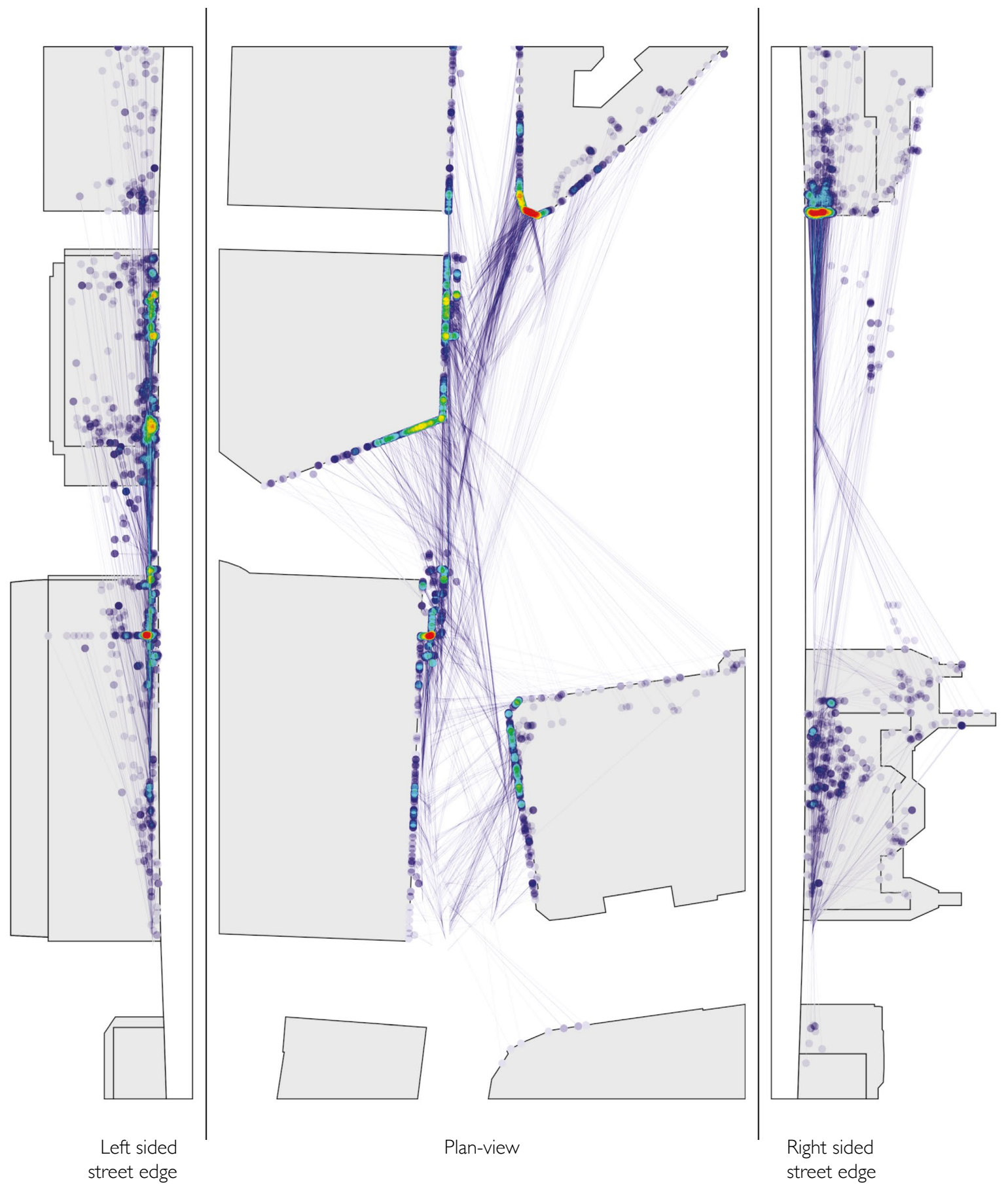

Heat-map key 
